\title{
Vulnerable and Resilient Cognitive Responses to Early-Life Stress: The Potential Mediating Role of Dopaminergic Receptors in the Prefrontal Cortex of Adult Mice
}

Saulo G. Tractenberg a, Rodrigo Orso ${ }^{\text {b }}$, Kerstin C. Creutzberg ${ }^{b}$, Luiza M. Malcona, Francisco S. Lumertz ${ }^{a}$, Luis Eduardo Wearick-Silva ${ }^{b}$, Thiago W. Viola ${ }^{b}$, Marco A. Riva ${ }^{c},{ }^{*}$ Rodrigo Grassi-Oliveira ${ }^{\mathrm{a}, \mathrm{b}}$

a Pontifical Catholic University of Rio Grande do Sul (PUCRS), School of Healthy Sciences, Developmental Cognitive Neuroscience Lab, Porto Alegre, RS, Brazil.

b Pontifical Catholic University of Rio Grande do Sul (PUCRS), Brain Institute (InsCer), Developmental Cognitive Neuroscience Lab, Porto Alegre, RS, Brazil.

c Università degli Studi di Milano, Department of Pharmacological and Biomolecular Sciences, Milan, Italy.

Authors email address: Saulo G. Tractenberg, saulogantes@gmail.com, Rodrigo Orso, rodrigo orso@hotmail.com, Kerstin C. Creutzberg, kerstin.creutzberg@acad.pucrs.br, Luiza M. Malcon, lzamalcon@gmail.com, Francisco S. Lummertz, fsindermannlumertz@gmail.com, Luis Eduardo Wearick-Silva, lewearick@gmail.com, Thiago W.Viola, thiago.viola@acad.pucrs.br, Marco A. Riva, m.riva@unimi.it, Rodrigo Grassi-Oliveira*

\section{Funding Statement}

This study was supported by grants [442776/2018-7] and [307130/2018-5] of RGO and [141182/2016-4] of SGT from Conselho Nacional de Desenvolvimento Científico e Tecnológico (CNPq) and [88881.189914/2018-01] of SGT from Coordenação de Aperfeiçoamento de Pessoal de Nível Superior (CAPES)

\section{Declarations of interest: none}

\section{Ethics approval statement}

This research was approved by the Ethics Committee on Animal Use (CEUA) of PUCRS, Brazil under the registration \#14/00421

\footnotetext{
*Corresponding author information:

Rodrigo Grassi-Oliveira, Avenida Ipiranga, 6681, predio 11, sala 928 - Porto Alegre, RS - Brazil, 90619-900. Email address: rodrigo.grassi@pucrs.br; ORCID ID: https://orcid.org/0000-0001-9911-5921
} 


\section{Abstract}

Rationale: Exposure to early-life stress (ELS) is known to have pronounced effects on the prefrontal cortex (PFC), which may lead to dysfunctions later in life. However, not all individuals exposed to ELS manifest the same negative neurobiological and behavioral phenotypes, and the mechanisms underlying the consequences of ELS are not fully understood. Dopamine signaling could be a key factor in understanding the effects of stress on PFC functionality and dysfunctions. Objectives: We aimed to investigate the differential effects of ELS on cognitive performance in adult mice and to examine the role of dopaminergic receptor expression in the PFC. Methods: BALB/c males were exposed to the maternal separation (MS) procedure and their cognitive performance on the eight-arm radial maze (8-RAM). For molecular-level assessments, we performed mRNA expression analyses for dopamine receptors-DRD1, DRD2, DRD3-and Hers1 expression in the PFC. Results: While MS produced an overall impairment on 8-RAM, the stressed animals could be divided in two groups on the basis of their performance in comparison with the controls: those with impaired cognitive performance (vulnerable to maternal separation, V-MS) and those that did not show any impairment (resilient to maternal separation, R-MS). V-MS animals also showed increased expression of DRD1 and DRD2 in comparison with R-MS animals and controls. Errors on 8RAM were also positively correlated with $D R D 1$ and $D R D 2$ mRNA expression. Conclusions: Our findings suggest a potential role of the dopaminergic system in the programming mechanisms of vulnerability and resilience to ELS. Alterations in dopamine receptor expression could represent a vulnerability factor in the response to ELS.

Keywords: Stress, Early-life Stress, Maternal Separation, Resilience, Working memory, Dopamine. 


\section{Introduction}

Adverse early-life experiences, such as childhood maltreatment and other stressor events, are known to be risk factors for the development of neuropsychiatric disorders later in life (Lupien, McEwen, Gunnar, \& Heim, 2009; Teicher \& Samson, 2013; Teicher, Samson, Anderson, \& Ohashi, 2016). One of the core aspects of this high-risk neuropsychiatric profile is related to the adverse effects of early-life stress (ELS) on neurodevelopment and the persistent structural and functional changes in central brain regions such as the prefrontal cortex (PFC) and the hippocampus (Hipp). These regions were commonly found to be significantly impaired in both human (MD, Hooper, Spratt, \& Woolley, 2009; Mothes et al., 2015; Navalta, Polcari, Webster, Boghossian, \& Teicher, 2006; Nikulina \& Widom, 2013; Oswald et al., 2014) and animal (Aisa et al., 2009; Chocyk et al., 2013; de Azeredo et al., 2017; Grassi-Oliveira, Honeycutt, Holland, Ganguly, \& Brenhouse, 2016; Reincke \& HanganuOpatz, 2017) studies, addressing the effects of ELS later in life.

Since the PFC is one of the brain regions that are most sensitive to the effects of ELS and pronounced cognitive deficits are observed in PFC-dependent tasks (Arnsten, 2009; Grassi-Oliveira et al., 2016; Viola et al., 2019), it is crucial to identify the potential mechanisms that can explain the adverse effects of stress on PFC functionality. One interesting hypothesis about PFC regulation is related to the role of dopamine signaling in specific networks and their output pathways, which affect distinct cognitive functions that are dependent on this brain region (e.g., working memory, attention, planning and solving problems, cognitive flexibility, decision-making, and goal-directed behaviors) (Holroyd \& Umemoto, 2016; Jenni, Larkin, \& Floresco, 2017; Kehagia, Murray, \& Robbins, 2010; Puig, Antzoulatos, \& Miller, 2014; Puig \& Miller, 2015; Winstanley \& Floresco, 2016). During cognitive tasks that require PFC recruitment, a significant release of endogenous dopamine (DA) as a consequence of DA neuron stimulation has been identified (Murphy, Arnsten, Goldman-Rakic, \& Roth, 1996; Ott 
\& Nieder, 2019; Vijayraghavan, Wang, Birnbaum, Williams, \& Arnsten, 2007). DA acts by binding to two classes of receptors, D1- and D2-like receptors, and their respective subdivisions (D1- and D5- receptors - DRD1 and DRD5- for the first class and D2-, D3-, and D4-receptors-DRD2, DRD3, and $D R D 4$-for the second class). DRD1 and DRD2 are considered the most abundant receptors expressed in the brain, and DA action on both of these receptors is important to facilitate PFC engagement (Araki, Sims, \& Bhide, 2007). Excessive stimulation of DA receptors in PFC, however, has been associated with deleterious effects on working memory functioning and other cognitive processes in an inverted- $U$ shape relation (Murphy et al., 1996; Vijayraghavan et al., 2007; Williams \& Castner, 2006).

A recent review and genome-wide studies (GWAS) (Knowles, Carless, et al., 2014; 2004) compiled the potential target genes associated with PFC functionality, especially in relation to working memory performance. In their findings it has been supported a mediating role of neuronal excitability through ion channel activity and/or dopaminergic signaling, highlighting the direct and indirect effects of DA expression in the PFC. Alterations in the excitability or efficiency of dopaminergic signaling were suggested to be involved in working memory dysfunction. Furthermore, Knowles (2014) found an interesting candidate gene, the basic helix-loop-helix (bHLH) transcriptional factor Hesrl gene (hairy and enhancer of splitrelated 1, also called $H E Y 1$ ), which has been shown to be involved in neurodevelopmental processes (e.g., neurogenesis and differentiation), neuropsychiatric disorders, and behavioral traits characterized by dysregulation of dopaminergic signaling. The Hesr 1 gene binds to 3' non-coding region of the dopamine transporter gene (DAT1), repressing the expression of $D A T 1$ and indirectly affects the expression of dopamine-related genes $(D 1, D 2, D 4, D 5)$ (Fuke et al. 2006). In Hesr 1 knockout mice, it was found an upregulation of DAT1, suggesting also a compensatory increase on dopamine receptor genes expression, which may be involved on 
working memory deficits and altered behavioral phenotype in classical behavioral tasks, such as open-field and elevated plus-maze (Fuke et al., 2006; Knowles, Mathias, et al., 2014).

Despite the significant data suggesting that ELS experiences lead to behavioral and cognitive dysfunctions in consequence of the abnormal neurodevelopment trajectory, it is difficult to infer a deterministic prediction. Inconsistent data among preclinical studies (Bonapersona, Joels, \& Sarabdjitsingh, 2018; Molet, Maras, Avishai-Eliner, \& Baram, 2014; Nylander \& Roman, 2013; Tractenberg et al., 2016), and the lack of knowledge regarding why some stressed individuals are susceptible to the negative consequences of early-life experiences while others seem to be "unaffected" or resilient seem to contribute to these mixed findings (Miller, Chen, \& Parker, 2011; Silk et al., 2007; Yehuda, Flory, Southwick, \& Charney, 2006). Some efforts have been made in this direction, particularly when distinct expression patterns of specific target-genes related to cognitive dysfunctions seen among subgroups of stressed animals were investigated. Studies addressing stress-reactive and non-stress reactive groups, or vulnerable and resilient groups, have advanced our knowledge of how distinctive ELS effects emerge and operate at the interface between gene-environmental factors (Calabrese et al., 2017; Clinton, Watson, \& Akil, 2014; Rana et al., 2016; Spencer-Segal \& Akil, 2019; Srinivasan, Shariff, \& Bartlett, 2013).

Here, we aimed to investigate the effects of ELS on working memory and memory function and on the expression target genes related to dopaminergic signaling (Hers 1, DRD1, $D R D 2$, and $D R D 3$ ) in the medial prefrontal cortex (mPFC). In addition, to improve our potential findings, we explored how animals subjected to ELS would differentially respond to stress exposure by using an experimental design that separated the stressed animals that showed a cognitive response to ELS from those that were resilient.

\section{Materials and methods}




\subsection{Animals}

All animals were purchased from the animal facility of the Center for Experimental Biological Models (CeMBE) at Pontifical Catholic University of Rio Grande do Sul (PUCRS), Brazil. Male BALB/c mice (total, $n=38$ ) were used for this experiment. All of them were housed in standard plastic mouse cages $(22 \mathrm{~cm} \times 16 \mathrm{~cm} \times 14 \mathrm{~cm}), 2-3$ animals per cage, and kept under constant housing and handling conditions (light/dark cycle: $12 \mathrm{~h}$, lights on at 6 a.m.; room temperature: $21^{\circ} \mathrm{C} \pm 1{ }^{\circ} \mathrm{C}$; humidity: $55 \% \pm 5 \%$; controlled air circulation; and, food and water regime ad libitum).

Breeding procedures involved housing two female mice with one male mice per cage during a mating period of two weeks. At the end of this period, the male was removed, and the females were individually housed and inspected daily at the same time until pregnancy confirmation. The day when pups were found was defined as postnatal day 1 (PND1). A crossfostering procedure was run with half of the litter, followed by litter control from 5 to 8 pups per litter, when necessary. Subsequently, the animals were randomly assigned to undergo prolonged maternal separation (MS group) or animal facility rearing (AFR group). For this experiment, we used a total of 18 different litters, of which 11 were designated for the MS group and 7 were for the AFR group. We used a total of $25 \mathrm{MS}$ animals (1-4 animals per litter) and 13 AFR (1-3 animals per litter). Surplus females were assigned to additional ongoing projects in the laboratory. Pups were not sexed until weaning day (PND21), preventing additional manipulations. At PND21, the males were weighed and group housed. Cage cleaning was performed once a week by the animal facility staff throughout the experimental period. The cognitive experimental procedures were assessed at early adulthood, from PND58 to PND67.

All procedures were approved by the Ethics Committee on Animal Use (CEUA) of PUCRS, Brazil under the registration \#14/00421. We conducted the experiments in accordance 
with National Institutes of Health guide for the care and use of Laboratory animals (NIH Publications No. 8023, revised 1978) and International Council for Laboratory Animal Science (ICLAS).

2.2 Maternal Separation (MS) procedures

MS procedures were performed following the methodological recommendations proposed in our extended MS systematic review (for more details, see Tractenberg et al., 2016) and well established by prior studies (Bailoo, Jordan, Garza, \& Tyler, 2014; Wang, Jiao, \& Dulawa, 2011). From PND2 to PND15, the pups of the MS group were separated daily from their dams for a period of 180 minutes, during the end period of the light cycle (4 p.m. to 7 p.m.). The procedure involved first removing the dams from the home cage and reallocating them to a new cage containing standard clean bedding. Dams were also transferred to another room to avoid ultrasonic vocalization between dams and the pups. Pups were then removed from the home cage and individually distributed in a small plastic box containing a little amount of bedding. The temperature of the pups was controlled by a digital-regulated heating pad (around $32^{\circ} \pm 3^{\circ} \mathrm{C}$ ) in order to prevent stress by hypothermia. After the 180 -min period, the pups were returned to their home cage followed by the dam. The animals in the AFR group was left undisturbed in their home cages throughout the postnatal period.

\subsection{Eight-arm radial maze test (8-RAM)}

To evaluate cognitive parameters related to working memory, visuospatial memory, and learning, we conducted an adapted version of the 8-RAM protocol (Ammassari-Teule, Hoffmann, \& Rossi-Arnaud, 1993; Umemori et al., 2013; Valentim, Olsson, \& Antunes, 2013; Valladolid-Acebes et al., 2011; Yadav et al., 2013), with four-arms baited, as first described by Jarrard (1983). The apparatus consisted of eight arms extending radially from a central 
octagonal platform $(5 \mathrm{~cm}$ wide $\mathrm{X} 30 \mathrm{~cm}$ long, extending from a central octagonal platform $25 \mathrm{~cm}^{2}$ ). Each of the eight arms had internal fixed visual clues put on the floor below the apparatus. The clues were prepared as described in Ammassari-Teule's study (1993) by using different visual patterns (e.g., black, black squares, white, longitudinal stripes). Each arm, numbered from 1 to 8 , had a specific visual pattern clue that was permanent throughout the experimental period. Additionally, four external clues were attached to the walls around the maze. The stimuli were shapes cut from black cardboard (e.g., a rectangle, a circle, a triangle, and a half moon). (Valladolid-Acebes et al., 2011). The overhead lights were on. We ran a 14day session broken into three phases: training (day 1-4), testing (day 5-13), and retention (day 14) explained in detail below. During all phases, the maze was kept in a constant orientation. The training phase occurred twice a day in the morning (9 a.m.) and in the afternoon (13 a.m.). Since our protocol depends on goal-directed behavior, we submitted the animals to a $20-\mathrm{h}$ hydric restriction condition, as previously suggested by Rowland (2007). Thus, every day at the end of the procedures, the animals were placed in an ad libitum water condition and all bottles of water were removed 4 h later, at 5 p.m. The hydric restriction condition started 1 week before the testing phase to acclimate the animals with this regimen and minimize stressinduced effects. We used as reward a diluted solution of $10 \%$ condensed milk, using $20 \mu \mathrm{L}$ of this solution as bait.

Training phase: On day 1, all animals of the same home cage were placed together on the center platform and allowed to explore the entire maze for $20 \mathrm{~min}$ in the morning time. Later, during the afternoon, the animals were placed individually and were allowed to explore all eight arms for $10 \mathrm{~min}$. At this time, all the arms were baited at the beginning and the ending; on day 2 , the day 1 afternoon procedure was repeated as described above; on day 3 , animals were placed individually on the central platform and allowed to explore all arms for $10 \mathrm{~min}$ or until all rewards were collected. All the arms were baited just at the ending (on the collecting 
area). The procedure was performed in the same form in the morning and in afternoon; on day 4, animals were placed individually and were free to explore all arms for $10 \mathrm{~min}$; however, at this time, just four of the eight arms were baited. If the animals retrieved all four rewards before the time ran out, the trial was finished, and the animal was removed from the maze. This procedure was performed twice on this day, in the morning and in the afternoon. The four baited arms were randomly assigned for each animal (e.g., 1-3-5-7 or 2-4-6-8) and were maintained throughout the testing phase.

Testing phase: During days 5-13, the procedure was run in the same way throughout the testing phase. This phase consisted of two consecutive trials per animal performed once a day (13 a.m.). The inter-trial interval was about 2 min. For each trial, all animals were placed individually on the center platform inside a transparent cylinder; the trial started when the cylinder was removed (5-s delay) and the animal was free to explore all arms for $10 \mathrm{~min}$ or until all rewards were retrieved. In order to eliminate or reduce olfactory cues, before each animal and between trials, the maze was wiped with a $70 \%$ ethanol solution.

Retention phase: At day 14, all animals were placed at the maze to run a single 10-min trial or until they collected all rewards.

The parameters evaluated for each trial were as follows: the time spent to collect all rewards; the number of arm entrances; the number of working memory errors - when the animal reentered a baited arm that had already been visited in that trial; the number of reference memory errors - number of entries in an arm that had never been baited; the number of perseverative errors - when the animal reentered an unbaited arm that had already been visited in that trial. An arm entry was defined when the animal was entirely (four paws and tail) inside the arm. A correct entry was defined as a first entry/reward consumed into a baited arm (Avdesh, Hoe, Martins, \& Martin-Iverson, 2013; Nozari, Mansouri, Shabani, Nozari, \& Atapour, 2015). All measures were registered by an observer while the experiment was 
running. We also calculated the sum of both trials of mentioned errors for each day. Following the data analyses of behavioral parameters, we used the sum of all 8-RAM errors to divide the MS group into two subgroups (those with significant deficits were considered vulnerable, VMS group and those that performed similar than controls, were considered resilient, R-MS group).

\subsection{Euthanasia and brain tissue collection}

All animals were euthanized by decapitation 30 min after the last trial of the testing phase. Trunk blood was immediately collected from the site where the animal is decapitated and stored in 1.5-mL Eppendorf tubes containing $\cong 100 \mu \mathrm{L}$ of ethylenediaminetetraacetic acid (EDTA) solution at room temperature until all euthanasia procedures were finished. Next, we centrifuged the samples at $1.000 \times g$ for $10 \mathrm{~min}$ at a temperature of $17^{\circ} \mathrm{C}$. The plasma was collected and stored at $-80^{\circ} \mathrm{C}$ until use. At the same time, the brains were removed and the $\mathrm{mPFC}$ region was dissected manually using the free-hand dissection method with tweezers and scalpel in the following bregma coordinates: anteroposterior from $1.34 \mathrm{~mm}$ to $1.98 \mathrm{~mm}$, mediolateral $\pm 0.5 \mathrm{~mm}$ and dorsoventral from $-1.0 \mathrm{~mm}$ to $-3.0 \mathrm{~mm}$. Following dissection, samples were immediately frozen in dry ice. All samples were stored at $-80^{\circ} \mathrm{C}$ until the day of molecular analysis.

\subsubsection{Plasma corticosterone (CORT) Assay}

Plasma was defreezed and the Corticosterone Enzyme Immunoassay (Arbor Assays) ELISA kit was used with $5 \mu \mathrm{L}$ of the sample in accordance with the manufacturer`s guidelines. From the total number of animals $(n=38)$ analyzed for obtaining behavioral data, 36 samples were used in CORT analysis, while 2 samples were lost during plasma extraction. From the data analyses, we excluded 5 outlier values considering the SPSS cutoff point (1.5XIQR, 
Interquartile range) (AFR: $n=3$ and V-MS: $n=2$ ). The optical density was determined at a wavelength of $450 \mathrm{~nm}$ in the ELISA plate reader. We subsequently transformed the value into $\mathrm{pg} / \mathrm{mL}$ concentrations using standard curve parameters.

\subsubsection{Transcript mRNA levels}

A total of 18 samples were used for mRNA analysis. $N=6$ samples from each group (V-MS, R-MS and AFR) were randomly selected and total RNA was isolated using QIAzol (Qiagen; Hilden, Germany) and chloroform standard protocols. The RNA concentration was measured using the NanoDrop spectrophotometer. Next, $1 \mu \mathrm{g}$ of RNA from each sample was reverse-transcribed using the miScript II RT Kit (Qiagen). The following primers (IDT) were used: Drdl (For: ATGGCTCCTAACACTTCTACCA, Rev: GGGTATTCCCTAAGAGAGTGGAC)，Drd2 (For：ACCTGTCCTGGTACGATGATG, Rev: GCATGGCATAGTAGTtGTAGTGG), $\quad \operatorname{Drd3}$ (For: CCTCTGAGCCAGATAAGCAGC , Rev: AGACCGTTGCCAAAGATGATG) and Hers 1 (For: GAAGCGCCGACGAGACCGAATCAA, Rev: CAGGGCGTGCGCGTCAAAATAACC). The SYBR Green PCR was performed in duplicate for each sample using a Rotor Gene Real-Time PCR machine (Qiagen). Fold-change relative expression was calculated through the $\Delta \Delta \mathrm{Ct}$ method with the AFR group as a reference. Probable phosphoglycerate kinase (Pgk): (For: TGCACGCTTCAAAAGCGCACG, Rev: AAGTCCACCCTCATCACGACCCAFR) values were used as the endogenous control for mRNA analysis. To verify primer specificities, melting curve analyses and agarose gel assessments were performed.

\subsection{Statistical analyses}


Normal distribution and homogeneity of variances were tested using the KolmogorovSmirnov test. All variables showed normality, and parametric analyses were assumed. The analyses were performed using Student's independent $t$-test to analyze the "total number of errors" between the MS and AFR groups; one-way analysis of variance (ANOVA) for dichotomization of groups and to compare the AFR, vulnerable to MS (V-MS) and resilient to MS (R-MS) groups; and repeated-measures ANOVA with the between-subjects factor "dichotomized groups" and the within-subject factor "days-block" for all evaluated 8-RAM parameters. The "days-block" variable was created using the mean score of the sum of 8-RAM parameters through three different intervals [block-1: $\Sigma$ (day 1 to 3$) / 3$; block-2: $\Sigma$ (day 4 to 6$) / 3$; and block-3: $\Sigma$ (day 7 to 9)/3]. Post-hoc analyses were adjusted with Turkey HSD correction. Pearson's correlation analyses were also used to examine possible associations between behavioral and biological parameters. $P$-values less than 0.05 were considered significant in our analyses. Values in graphs are expressed as the mean \pm SEM. All results were explored and analyzed by SPSS 21.0 for Windows (SPSS Inc., Chicago, IL, USA). Graphs were generated using GraphPad Prism 7 (GraphPad Software Inc., La Jolla, CA, USA).

\section{Results}

\subsection{Group dichotomization}

We created a variable based on the sum of all 8-RAM errors (working memory error, perseverative errors, and reference errors). The average number of total errors in the control group (AFR) $(n=13)$ was $93.61 \pm 12.29$ while that in the MS group $(n=25)$ was $146.76 \pm$ 37.71, which was statistically different $(t=-6.42, d f=32.12, p<0.0001)$ (Fig. 1a). Based on the cutoff point of three standard deviations from the mean (AFR group $=93.61 \pm 12.29$ ), our cutoff point was set as 131 . MS animals with $>131$ total errors were considered as V-MS and those with $<131$ total errors were considered as R-MS. Significant differences between groups 
were identified considering the number of total errors $(F=63.77, d f=2,35, p<0.0001)$. Posthoc test comparisons revealed significant differences between the V-MS and R-MS groups ( $p$ $<0.0001)$ and V-MS and AFR groups $(p<0.0001)$. No differences were found between the RMS and AFR groups ( $p=0.1681)$ (Fig. 1b). The percentage of animals who met the criteria within each group is shown in Figure 1c. $N=15$ animals met the criterion for the V-MS group while $n=10$ met the criterion for the R-MS group.

\section{INSERT FIGURE 1 HERE}

3.2 V-MS versus R-MS behavioral analyses:

ANOVA showed significant intergroup differences for different parameters of the 8RAM in both testing and retention phases (Fig. 2). The total number of working memory errors (WMEs) was significantly different $(F=38.80, d f=2,35, p<0.0001)$. Post-hoc analysis revealed significant differences between the V-MS and both R-MS and AFR groups ( $V$ - $M S$ vs. $R-M S, p<0.0001$ and $V$ - $M S$ vs. $A F R, p<0.0001)$. No difference was found between the RMS and AFR groups ( $p=0.3997)$ (Fig. 2a). The total number of perseverative errors (PSEs) was also significantly different $(F=44.09, d f=2,35, p<0.0001)$. A post-hoc test showed differences between V-MS and both R-MS and AFR groups ( $V$ - $M S$ vs. $R, p<0.0001$ and $V$ $M S$ vs. $A F R, p<0.0001)$. No difference was found between the R-MS and AFR groups ( $p=$ 0.1803) (Fig. 2b). The total number of reference errors (REs) was significantly different $(F=$ 14.85, $d f=2,35, p<0.0001)$. Post-hoc analysis revealed differences between the V-MS group and both R-MS and AFR groups ( $V-M S$ vs. $R-M S, p<0.0022$ and $V-M S$ vs. $A F R, p<0.0001)$. No difference was found between the R-MS and AFR groups $(p=0.5127)$ (Fig. 2c). The number of retention working memory errors ( $\mathrm{r}$-WMEs) was significantly different $(F=5.69$, $d f=2,35, p<0.0073)$. Post-hoc analysis revealed differences between the V-MS group and 
both R-MS and AFR groups ( $V-M S$ vs. $R-M S, p<0.0427$ and $V-M S$ vs. $A F R=, p<0.0109)$. No difference was found between the R-MS and AFR groups ( $p=0.7337$ ) (Fig. 2d). Moreover, the number of retention perseverative errors (r-PSEs) was also significantly different $(F=4.07$, $d f=2,35, p<0.0256)$. Post-hoc analysis revealed differences between the V-MS and R-MS groups ( $V-M S$ vs. $R-M S, p<0.0301)$. No difference was found between the AFR group and both V-MS ( $p=0.1115)$ and R-MS ( $p=0.7482)$ groups (Fig. 2e). Lastly, the number of retention reference errors (r-REs) failed to reveal significant differences among the three groups $(p=0.0831)$ (Fig. 2f).

\section{INSERT FIGURE 2 HERE}

\subsection{Days-block analyses:}

Block analysis was conducted in order to explore how the animals performed over three different phases of the task and to evaluate the transition from goal-directed behavior to habitdirected behavior. Analyses of the time spent to complete the task (TIME), WMEs, PSEs, and REs during the testing phase at block-1, block-2, and block-3 using repeated-measures oneway ANOVA revealed a significant block effect (TIME: $[F=19.63, d f=2,70, p<0.0001$, post-hoc]; WME: $[F=8.95, d f=2,70, p<0.0001]$; PSE: $[F=22.8, d f=2,70, p<0.0001]$; $\operatorname{RE}[F=9.48, d f=2,70, p<0.0001])$ and group effect (TIME: $[F=13.16, d f=2,70, p<$ 0.0001]; WME: $[F=38.8, d f=2,35, p<0.0001]$; PSE: $[F=36.2, d f=2,35, p<0.0001]$; RE $[F=14.85, d f=2,35, p<0.0001])$. No interaction of block $\mathrm{X}$ group was found for all parameters (Fig. 3). Post-hoc analysis for block effect showed significant differences in TIME (block-1 $>$ block-2, $p<0.0301$ and block $-3, p<0.0001$ and block-2 $>$ block-3, $p<0.0009$ ), revealing that animals were able to reduce the time spent to complete the task across the trialblocks, and significant differences in WME (block-1 > block-3, $p<0.0045$ ); PSE (block-1 > 
block-2, $p<0.0033$ and block-3, $p<0.0001$ and block- $2>$ block-3, $p<0.0134$ ) and RE (block$1>$ block-2, $p<0.0466$ and block-3, $p<0.0004)$, indicating that animals were able to reduce the number of errors from the block-1 to block-3. Post-hoc analysis for group effect showed significant differences in TIME (V-MS $>$ R-MS, $p<0.0002$ and V-MS $>$ AFR, $p<0.0013$ ), revealing that V-MS group spent more time to complete the task compared to R-MS and AFR groups, and significant differences in WME (V-MS $>$ R-MS $p<0.0001$ and V-MS $>$ AFR, $p$ $<0.0001)$; PSE (V-MS > R-MS $p<0.0001$ and V-MS > AFR, $p<0.0001)$ and RE (V-MS > R-MS $p<0.0071$ and V-MS $>$ AFR, $p<0.0001$ ), indicating that V-MS group had higher number of errors than R-MS and AFR groups.

\section{INSERT FIGURE 3 HERE}

\section{$3.4 V-M S \times R-M S$ molecular analyses:}

Next, we investigated the plasma levels of corticosterone (CORT) as a proxy for hypothalamic pituitary adrenal (HPA) activity. Our comparative analysis revealed a significant difference between groups $(F=7.83, d f=2,32, p<0.0002)$. Post-hoc analysis showed that CORT levels were significantly increased in the R-MS group, when compared to the AFR group $(R-M S$ vs. $A F R, p<0.0013)$ No statistical differences were observed between the V-MS and AFR ( $p=0.0915)$ or R-MS ( $p=0.1128)$ groups (Fig. 4). Our transcriptional analyses found significant differences between groups with respect to the mRNA levels of DRDI $(F=5.09$, $d f=2,17, p<0.0218)$ and DRD2 $(F=11.51, d f=2,17, p<0.0012)$. Post-hoc analysis revealed a significant increase in $D R D 1$ mRNA levels in the V-MS group, in comparison with the R-MS group ( $V$ - $M S$ vs. $R-M S, p<0.0221$ ), and in $D R D 2$ mRNA expression, in comparison with both R-MS and AFR groups ( $V$-MS vs. $R-M S, p<0.0038$ and $V$-MS vs. $A F R, p<0.0016)$. 
No differences among the experimental groups were found for DRD3 $(p=0.640)$ and Hers 1 $(p=0.6688)$ mRNA levels (Fig. 5).

\section{INSERT FIGURE 4 AND 5 HERE}

3.4 Correlational analyses:

Finally, we performed Pearson correlation analyses with all 8-RAM parameters (WMEs, PSEs, and REs) in both testing and retention phases, plasma CORT levels, and the mRNA levels for DRD1, DRD2, DRD3 and Hers1. All correlations are presented in Table 1. We found a significant positive correlation between both mRNA DRD1 and DRD2 for WME and PSE errors (Fig. 6). mRNA DRDl was positively correlated with WME errors $(\mathrm{r}=0.53, p$ $<0.0216)$ and PSE errors ( $\mathrm{r}=0.53, p<0.0234)$ (Fig. 6a) and mRNA DRD2 was also positively correlated with WME errors $(\mathrm{r}=0.51, p<0.0291)$ and PSE errors $(\mathrm{r}=0.61, p<0.0067)($ Fig. $6 b)$.

\section{INSERT TABLE 1 AND FIGURE 6 HERE}

\section{Discussion}

This study investigated the effects of MS on working memory functioning and on DA receptors (DRD1, DRD2 and DRD3) and Hers 1 mRNA expression in the mPFC, assessing the potential differences among maternally separated animals, since we were interested in determining how these animals could be differentially affected by ELS. Our findings revealed that, as expected, MS led to impaired working memory and reference memory in 8-RAM considering the number of total errors committed along all testing days. This result is consistent with prior studies showing that different ELS models can disrupt working memory and memory 
processes (i.e., consolidation and retrieval processes), through functional alterations of specific brain regions (Cattaneo et al., 2019; Chocyk et al., 2013; de Azeredo et al., 2017; GrassiOliveira et al., 2016; Jin et al., 2013; Liu, Atrooz, Salvi, \& Salim, 2017; Mehta \& Schmauss, 2011; van der Kooij, Grosse, Zanoletti, Papilloud, \& Sandi, 2015). However, not all MS animals presented the same pattern of cognitive performance. In fact, there was a specific subgroup of MS animals that showed similar performance as controls, while another subgroup showed worse performance on the task.

Differences in stress responses between subjects exposed to stress paradigms have already been demonstrated by some studies (Aydin, Frohmader, \& Akil, 2015; Calabrese et al., 2017; Clinton et al., 2014; Rana et al., 2016; Spencer-Segal \& Akil, 2019) and considering our data for distinct patterns of cognitive performance within the MS group, we focused on comparisons between the responsive and non-responsive MS animals $(\mathrm{V}-\mathrm{MS} \times \mathrm{R}-\mathrm{MS})$ for all parameters of 8-RAM. Our data revealed that for all parameters of 8-RAM (WMEs, PSEs, and REs), the V-MS group had a higher number of errors compared to the R-MS and control groups. The same results were found for the retention test ( $24 \mathrm{~h}$ after), except for retention reference errors. Although V-MS animals were able to learn and reduce the number of errors and time spent to collect the rewards over the testing period, showing an improvement of their performance along the trials, the V-MS animals performed worse than resilient and control animals. This indicated that V-MS generally still showed significant impairment on working memory processes compared to other groups. Such deficits probably narrow the ability to store essential information for learning and to move from goal-directed behavior to habit-directed behavior (Puig et al., 2014; Wickens, Horvitz, Costa, \& Killcross, 2007), especially because they had higher number of perseverative errors. Thus, collectively, our findings indicate that not all maternally separated animals were equally affected by the stressor early in life, 
suggesting a discriminative cognitive phenotype between vulnerable and resilient subgroups for ELS effects.

Because the biomolecular targets underlying the distinct patterns of response to early stress exposure are not fully elucidated, we decided to investigate the potential mechanisms that may contribute to the differential cognitive outcomes observed in V-MS and R-MS mice. We investigated the mRNA levels of DA receptors (DRD1,DRD2 and DRD3) and Hers1. Our findings revealed alterations in DA receptors mRNA expression, especially in DRDI and DRD2. DRD1 and DRD2 mRNA expression were found higher in V-MS groups compared to the R-MS or AFR groups. Our correlational analysis revealed associations between different types of task-errors (WMEs and PSEs) and DRD1 and DRD2 mRNA expression, corroborating the idea that DA receptors expression could regulate cognitive performance (Knowles, Mathias, et al., 2014; Murphy et al., 1996). Both DRD1 and DRD2 have been suggested as important contributors to several cognitive functions, including working memory, decision making, cognitive flexibility, and learning processes under certain circumstances (Puig et al., 2014). Regarding their action on memory function, for example, it was demonstrated that both are essential to initial phases of learning processes and for transition from goal-directed behavior to habit-directed behavior during tasks with multiple trials (Mohebi et al., 2019; Puig et al., 2014; Vijayraghavan et al., 2007).

The overexpression of DA receptors in PFC and PFC-related networks has been suggested as a potential contributor for the inverted-U shape relationship between dopaminergic transmission and cognitive efficiency (Vijayraghavan et al., 2007). An excessive increase of activity in dopaminergic system was suggested as able to negatively impact PFCdependent cognitive functioning (Azadmarzabadi, Haghighatfard, \& Mohammadi, 2018; Murphy et al., 1996; Vijayraghavan et al., 2007). When DRD1 agonists was administered in animals during working memory task, for example, it was observed suppressive effects on 
dopaminergic neuronal firing in PFC in association with performance deficits (Vijayraghavan et al., 2007). Altered behavior phenotype was also found in a recent study from our lab (Wearick-Silva et al., 2019) in association with upregulation of DRD1 in animals exposed to ELS through Limited Bedding protocol. It suggests that stress negative effects could be shape the brain development through dopamine system contributing for behavioral dysfunctional outcomes later in life. Thus, the remarkable deficits on working memory, cognitive flexibility, and memory retention found in V-MS animals on 8-RAM could be related to DRD1 and DRD2 mRNA overexpression in response to ELS exposure. All these evidences of DA receptors expression changes and its relationship with behavioral and cognitive deficits could highlight the effects of potential genetic variants and/or epigenetic modifications in DA pathways, supporting potentials biomolecular markers of vulnerability characterized by DA expression (Vijayraghavan et al., 2007).

In addition, animal data (Fuke et al., 2006; Jenni et al., 2017; Puig \& Miller, 2015; Vijayraghavan et al., 2007) have been found depletion on Hersl gene expression associated with increased DAT and DA receptors gene expression. This upregulation response as consequence of Hersl changes could influence the sensitivity of DA transmission, affecting the animal's behavior on cognitive tasks (Fuke et al., 2006; Knowles, Mathias, et al., 2014). In our study, however, we did not find any differences between groups to substantiate the prior findings regarding the role of Hersl in DA receptor expression imbalance. Only DRDI and DRD2 mRNA expression were found upregulated. Decreased DA concentrations in synaptic transmission, for example, are able to disrupt signaling transmission, provoking a regulatory response at DA receptor level, which could explain an upregulation of DA receptors gene expression (Fuke et al., 2006; Vijayraghavan et al., 2007).

The overactivation of DA receptors in the PFC combined with activation of stressinduced hormonal response, such as an increase in CORT levels following a stress exposure, 
also have been associated with working memory impairments (Garrido et al., 2013; Park et al., 2006; Williams \& Castner, 2006). In our study, unexpectedly, CORT levels were significantly increased in the R-MS group and no correlation was found between CORT levels and DA receptor mRNA levels. Altered HPA response and glucocorticoid levels were identified among ELS studies, supporting the negative effects of stress factors during the early periods of neurodevelopment (Anacker, O'Donnell, \& Meaney, 2014; Cohen et al., 2006; Frodl \& O'Keane, 2013; Yehuda et al., 2010). Acute and chronic exposure to glucocorticoids produces distinct effects on the organisms. A short-term increase in glucocorticoid levels has already been demonstrated as a neurobiological positive adaptation associated with reduction of dysfunctional anxiety-like behavior response, promoting resilience (Spencer-Segal \& Akil, 2019). In addition, CORT administration in rats before stress-induced tasks was associated with attenuation of anxiety and defensive response (Zohar et al., 2011), whereas a blunted CORT response has been related to an inadequate behavioral response (Cohen et al., 2006; Danan, Matar, Kaplan, Zohar, \& Cohen, 2018). This could be the case of our R-MS animals that showed an elevation of CORT levels following the cognitive task, considering the fact that our analyses were carried out 30 min after the last day of testing, Elevations in CORT levels in R-MS animals might promote certain cognitive performances, although the control animals showed lower CORT levels. As our V-MS responded differently, it could represent an abnormal acute stress-induce response and, consequently, be deleterious for cognitive adaptation to task demand. Because our study did not access basal CORT levels before the test beginning, it is difficult to establish how both maternally separated animals would chronically respond to stress, narrowing the extent of our conclusions.

Finally, it is important to note that our findings should be interpreted with some limitations. We based our cognitive evaluation in only 8-RAM at early adulthood and could not extend the conclusions to other cognitive domains and/or developmental time-point. 
However, the 8-RAM is considered a complex cognitive task that has been used in studies with rodents to evaluate multiple parameters (e.g., working memory, cognitive flexibility, spatial reference memory and learning) (Churchwell \& Kesner, 2011; Dudchenko, 2004), most of them recruiting more or less the activity of both $\mathrm{mPFC}$ and hippocampus. It is difficult to assume that this task is specific for evaluation of either PFC or hippocampus functionality, but some authors have been proposing a prominent role of one or another brain region depending on the type of error committed by the animal (Bilkey \& Liu, 2000; Yoon, Okada, Jung, \& Kim, 2008). For example, when the animals are trained (thought spatial stimulus) to make a choice response to get the reward and, after, have to keep this information over the time, a working memory demand are imposed. PFC play a critical role for attend this demand, while hippocampus is suggested to be more implicated in the learning and retrieval processes along the testing days (Yoon et al., 2008). It seems that both brain regions influence the performance but in different temporal domains, then damage to either structure are able to produce impairments. Studies in this line have been providing conclusions that instead a structural specificity, a functional interaction between both is required to perform this task (Matzel \& Kolata, 2010; Reincke \& Hanganu-Opatz, 2017). Further, we just investigate molecular targets looking at the gene expression layer. Although it represents an interesting exploratory screening, investigations using other techniques, (e.g., Western blotting and microdialysis) may be useful to address the proteins at cell-type level and the levels of dopamine while mice are performing the task, respectively, to better determine the function of the studied brain areas. To improve the cause-effect relationship studies should also explore specific receptor antagonists or agonists effects on dopaminergic function. In the same line, using a free-hand technique for brain dissection, we can not be precise at subregion analyses level, which should be considered when generalizing the molecular results. 


\section{Conclusion}

Our results showed that V-MS had poor performance on 8-RAM and that such cognitive deficits were associated with significant changes in the DA receptor expression. We suggest that dopaminergic signaling in PFC could be a key player in the cognitive dysfunctions associated with this brain region in response to ELS exposure. Future studies should focus on improving our understanding of the role of dopamine signaling in cognitive and behavioral response to ELS, addressing potential biomolecular markers that could better elucidate how

ELS affects dopaminergic signaling functionality during development and represent a susceptibility factor to neuropsychiatric diseases. In addition, such methodological approach could provide new insights regarding individual potential neurobiological distinctions than can bias the subjects toward vulnerability and resilience. Here, as an example, we highlighted the role of dopaminergic receptor expression changes in animals that revealed a vulnerable cognitive phenotype on a PFC-dependent task. 


\section{Statement of Contribution of all authors}

SGT, LM and FSL realized stress experimental procedures. SGT, RO, KCC ran 8-RAM protocol. KCC, LWS and TWV ran and interpreted biomolecular analyses. SGT, RO, and RGO were the major contributors in writing and TWV, MAR and RGO revised the final version of the manuscript. All authors declared that they read and approve manuscript final version.

\section{Acknowledgments}

We would like to thank Emerson S. Hoffmann who helped in initiating the experimental procedures during his period at the Developmental Cognitive Neuroscience Lab.

\section{Data Availability Statement}

The data that support the findings of this study are available from the corresponding author upon reasonable request. 


\section{References}

Aisa, B., Elizalde, N., Tordera, R., Lasheras, B., Del Río, J., \& Ramírez, M. J. (2009). Effects of neonatal stress on markers of synaptic plasticity in the hippocampus: implications for spatial memory. Hippocampus, 19(12), 1222-1231. doi:10.1002/hipo.20586

Ammassari-Teule, M., Hoffmann, H. J., \& Rossi-Arnaud, C. (1993). Learning in inbred mice: strain-specific abilities across three radial maze problems. Behav Genet, 23(4), 405412. Retrieved from https://www.ncbi.nlm.nih.gov/pubmed/8240221

Anacker, C., O'Donnell, K. J., \& Meaney, M. J. (2014). Early life adversity and the epigenetic programming of hypothalamic-pituitary-adrenal function. Dialogues Clin Neurosci, 16(3), 321-333. Retrieved from https://www.ncbi.nlm.nih.gov/pubmed/25364283

Araki, K. Y., Sims, J. R., \& Bhide, P. G. (2007). Dopamine receptor mRNA and protein expression in the mouse corpus striatum and cerebral cortex during pre- and postnatal development. Brain Res, 1156, 31-45. doi:10.1016/j.brainres.2007.04.043

Arnsten, A. F. (2009). Stress signalling pathways that impair prefrontal cortex structure and function. Nat Rev Neurosci, 10(6), 410-422. doi:10.1038/nrn2648

Avdesh, A., Hoe, Y., Martins, R. N., \& Martin-Iverson, M. T. (2013). Pharmacological effects of cannabinoids on the reference and working memory functions in mice. Psychopharmacology (Berl), 225(2), 483-494. doi:10.1007/s00213-012-2834-6

Aydin, C., Frohmader, K., \& Akil, H. (2015). Revealing a latent variable: individual differences in affective response to repeated injections. Behav Neurosci, 129(5), 679682. doi: $10.1037 / \mathrm{bne} 0000084$

Azadmarzabadi, E., Haghighatfard, A., \& Mohammadi, A. (2018). Low resilience to stress is associated with candidate gene expression alterations in the dopaminergic signalling pathway. Psychogeriatrics, 18(3), 190-201. Retrieved from https://onlinelibrary.wiley.com/doi/abs/10.1111/psyg.12312. doi:10.1111/psyg.12312 
Bailoo, J. D., Jordan, R. L., Garza, X. J., \& Tyler, A. N. (2014). Brief and long periods of maternal separation affect maternal behavior and offspring behavioral development in C57BL/6 mice. Dev Psychobiol, 56(4), 674-685. doi:10.1002/dev.21135

Bilkey, D. K., \& Liu, P. (2000). The effects of separate and combined perirhinal and prefrontal cortex lesions on spatial memory tasks in the rat. Psychobiology, 28(1), 12-20. Retrieved from https://doi.org/10.3758/BF03330625. doi:10.3758/bf03330625

Bonapersona, V., Joels, M., \& Sarabdjitsingh, R. A. (2018). Effects of early life stress on biochemical indicators of the dopaminergic system: A 3 level meta-analysis of rodent studies. Neurosci Biobehav Rev, 95, 1-16. doi:10.1016/j.neubiorev.2018.09.003

Calabrese, F., Brivio, P., Gruca, P., Lason-Tyburkiewicz, M., Papp, M., \& Riva, M. A. (2017). Chronic Mild Stress-Induced Alterations of Local Protein Synthesis: A Role for Cognitive Impairment. ACS Chem Neurosci, 8(4), 817-825. doi:10.1021/acschemneuro.6b00392

Cattaneo, A., Begni, V., Malpighi, C., Cattane, N., Luoni, A., Pariante, C., \& Riva, M. A. (2019). Transcriptional Signatures of Cognitive Impairment in Rat Exposed to Prenatal Stress. Mol Neurobiol, 56(9), 6251-6260. doi:10.1007/s12035-019-1523-4

Chocyk, A., Bobula, B., Dudys, D., Przyborowska, A., Majcher-Maslanka, I., Hess, G., \& Wedzony, K. (2013). Early-life stress affects the structural and functional plasticity of the medial prefrontal cortex in adolescent rats. Eur J Neurosci, 38(1), 2089-2107. doi:10.1111/ejn.12208

Churchwell, J. C., \& Kesner, R. P. (2011). Hippocampal-prefrontal dynamics in spatial working memory: interactions and independent parallel processing. Behav Brain Res, 225(2), 389-395. Retrieved from https://www.ncbi.nlm.nih.gov/pubmed/21839780. doi:10.1016/j.bbr.2011.07.045 
Clinton, S. M., Watson, S. J., \& Akil, H. (2014). High novelty-seeking rats are resilient to negative physiological effects of the early life stress. Stress, 17(1), 97-107. doi:10.3109/10253890.2013.850670

Cohen, H., Zohar, J., Gidron, Y., Matar, M. A., Belkind, D., Loewenthal, U., . . Kaplan, Z. (2006). Blunted HPA axis response to stress influences susceptibility to posttraumatic stress response in rats. Biol Psychiatry, 59(12), 1208-1218. doi:10.1016/j.biopsych.2005.12.003

Danan, D., Matar, M. A., Kaplan, Z., Zohar, J., \& Cohen, H. (2018). Blunted basal corticosterone pulsatility predicts post-exposure susceptibility to PTSD phenotype in rats. Psychoneuroendocrinology, 87, 35-42. doi:10.1016/j.psyneuen.2017.09.023

Dudchenko, P. A. (2004). An overview of the tasks used to test working memory in rodents. Neurosci Biobehav Rev, 28(7), 699-709. Retrieved from https://www.ncbi.nlm.nih.gov/pubmed/15555679.

doi:10.1016/j.neubiorev.2004.09.002

de Azeredo, L. A., Wearick-Silva, L. E., Viola, T. W., Tractenberg, S. G., Centeno-Silva, A., Orso, R., . . . Grassi-Oliveira, R. (2017). Maternal separation induces hippocampal changes in cadherin-1 (CDH-1) mRNA and recognition memory impairment in adolescent mice. Neurobiol Learn Mem, 141, 157-167. doi:10.1016/j.nlm.2017.04.006

Frodl, T., \& O'Keane, V. (2013). How does the brain deal with cumulative stress? A review with focus on developmental stress, HPA axis function and hippocampal structure in humans. Neurobiol Dis, 52, 24-37. doi:10.1016/j.nbd.2012.03.012

Fuke, S., Minami, N., Kokubo, H., Yoshikawa, A., Yasumatsu, H., Sasagawa, N., . . Ishiura, S. (2006). Hesr1 knockout mice exhibit behavioral alterations through the dopaminergic nervous system. J Neurosci Res, 84(7), 1555-1563. doi:10.1002/jnr.21062 
Garrido, P., De Blas, M., Ronzoni, G., Cordero, I., Anton, M., Gine, E., . . Mora, F. (2013). Differential effects of environmental enrichment and isolation housing on the hormonal and neurochemical responses to stress in the prefrontal cortex of the adult rat: relationship to working and emotional memories. J Neural Transm (Vienna), 120(5), 829-843. doi:10.1007/s00702-012-0935-3

Grassi-Oliveira, R., Honeycutt, J. A., Holland, F. H., Ganguly, P., \& Brenhouse, H. C. (2016). Cognitive impairment effects of early life stress in adolescents can be predicted with early biomarkers: Impacts of sex, experience, and cytokines. Psychoneuroendocrinology, 71, 19-30. doi:10.1016/j.psyneuen.2016.04.016

Holroyd, C. B., \& Umemoto, A. (2016). The research domain criteria framework: The case for anterior cingulate cortex. Neurosci Biobehav Rev, 71, 418-443. doi:10.1016/j.neubiorev.2016.09.021

Jarrard, L. E. (1983). Selective hippocampal lesions and behavior: effects of kainic acid lesions on performance of place and cue tasks. Behav Neurosci, 97(6), 873-889. Retrieved from https://www.ncbi.nlm.nih.gov/pubmed/6651962

Jenni, N. L., Larkin, J. D., \& Floresco, S. B. (2017). Prefrontal Dopamine D1 and D2 Receptors Regulate Dissociable Aspects of Decision Making via Distinct Ventral Striatal and Amygdalar Circuits. J Neurosci, 37(26), 6200-6213. doi:10.1523/JNEUROSCI.003017.2017

Jin, F., Li, L., Shi, M., Li, Z., Zhou, J., \& Chen, L. (2013). The longitudinal study of rat hippocampus influenced by stress: early adverse experience enhances hippocampal vulnerability and working memory deficit in adult rats. Behav Brain Res, 246, 116-124. doi:10.1016/j.bbr.2013.02.029 
Kehagia, A. A., Murray, G. K., \& Robbins, T. W. (2010). Learning and cognitive flexibility: frontostriatal function and monoaminergic modulation. Curr Opin Neurobiol, 20(2), 199-204. doi:10.1016/j.conb.2010.01.007

Knowles, E. E., Carless, M. A., de Almeida, M. A., Curran, J. E., McKay, D. R., Sprooten, E., ... Glahn, D. C. (2014). Genome-wide significant localization for working and spatial memory: Identifying genes for psychosis using models of cognition. Am J Med Genet B Neuropsychiatr Genet, 165B(1), 84-95. doi:10.1002/ajmg.b.32211

Knowles, E. E., Mathias, S. R., McKay, D. R., Sprooten, E., Blangero, J., Almasy, L., \& Glahn, D. C. (2014). Genome-Wide Analyses of Working-Memory Ability: A Review. Curr Behav Neurosci Rep, 1(4), 224-233. doi:10.1007/s40473-014-0028-8

Liu, H., Atrooz, F., Salvi, A., \& Salim, S. (2017). Behavioral and cognitive impact of early life stress: Insights from an animal model. Prog Neuropsychopharmacol Biol Psychiatry, 78, 88-95. doi:10.1016/j.pnpbp.2017.05.015

Lupien, S. J., McEwen, B. S., Gunnar, M. R., \& Heim, C. (2009). Effects of stress throughout the lifespan on the brain, behaviour and cognition. Nat Rev Neurosci, 10(6), 434-445. doi:10.1038/nrn2639

Matzel, L. D., \& Kolata, S. (2010). Selective attention, working memory, and animal intelligence. Neurosci Biobehav Rev, 34(1), 23-30. Retrieved from https://www.ncbi.nlm.nih.gov/pubmed/19607858.

doi:10.1016/j.neubiorev.2009.07.002

MD, D. E. B., Hooper, S. R., Spratt, E. G., \& Woolley, D. P. (2009). Neuropsychological findings in childhood neglect and their relationships to pediatric PTSD. J Int Neuropsychol Soc, 15(6), 868-878. doi:10.1017/S1355617709990464

Mehta, M., \& Schmauss, C. (2011). Strain-specific cognitive deficits in adult mice exposed to early life stress. Behav Neurosci, 125(1), 29-36. doi:10.1037/a0021952 
Miller, G. E., Chen, E., \& Parker, K. J. (2011). Psychological stress in childhood and susceptibility to the chronic diseases of aging: moving toward a model of behavioral and biological mechanisms. Psychol Bull, 137(6), 959-997. doi:10.1037/a0024768

Mohebi, A., Pettibone, J. R., Hamid, A. A., Wong, J. T., Vinson, L. T., Patriarchi, T., . . Berke, J. D. (2019). Dissociable dopamine dynamics for learning and motivation. Nature, 570(7759), 65-70. doi:10.1038/s41586-019-1235-y

Molet, J., Maras, P. M., Avishai-Eliner, S., \& Baram, T. Z. (2014). Naturalistic rodent models of chronic early-life stress. Dev Psychobiol, 56(8), 1675-1688. doi:10.1002/dev.21230

Mothes, L., Kristensen, C. H., Grassi-Oliveira, R., Fonseca, R. P., de Lima Argimon, I. I., \& Irigaray, T. Q. (2015). Childhood maltreatment and executive functions in adolescents. Child and Adolescent Mental Health, 20(1), 56-62. doi:10.1111/camh.12068

Murphy, B. L., Arnsten, A. F., Goldman-Rakic, P. S., \& Roth, R. H. (1996). Increased dopamine turnover in the prefrontal cortex impairs spatial working memory performance in rats and monkeys. Proc Natl Acad Sci U S A, 93(3), 1325-1329. doi:10.1073/pnas.93.3.1325

Navalta, C. P., Polcari, A., Webster, D. M., Boghossian, A., \& Teicher, M. H. (2006). Effects of childhood sexual abuse on neuropsychological and cognitive function in college women. J Neuropsychiatry Clin Neurosci, 18(1), 45-53. doi:10.1176/jnp.18.1.45

Neve, K. A., Seamans, J. K., \& Trantham-Davidson, H. (2004). Dopamine receptor signaling. J Recept Signal Transduct Res, 24(3), 165-205. Retrieved from https://www.ncbi.nlm.nih.gov/pubmed/15521361

Nikulina, V., \& Widom, C. S. (2013). Child maltreatment and executive functioning in middle adulthood: a prospective examination. Neuropsychology, 27(4), 417-427. doi:10.1037/a0032811 
Nozari, M., Mansouri, F. A., Shabani, M., Nozari, H., \& Atapour, N. (2015). Postnatal MK801 treatment of female rats impairs acquisition of working memory, but not reference memory in an eight-arm radial maze; no beneficial effects of enriched environment. Psychopharmacology (Berl), 232(14), 2541-2550. doi:10.1007/s00213-015-3890-5

Nylander, I., \& Roman, E. (2013). Is the rodent maternal separation model a valid and effective model for studies on the early-life impact on ethanol consumption? Psychopharmacology (Berl), 229(4), 555-569. doi:10.1007/s00213-013-3217-3

Oswald, L. M., Wand, G. S., Kuwabara, H., Wong, D. F., Zhu, S., \& Brasic, J. R. (2014). History of childhood adversity is positively associated with ventral striatal dopamine responses to amphetamine. Psychopharmacology (Berl), 231(12), 2417-2433. doi:10.1007/s00213-013-3407-z

Ott, T., \& Nieder, A. (2019). Dopamine and Cognitive Control in Prefrontal Cortex. Trends Cogn Sci, 23(3), 213-234. doi:10.1016/j.tics.2018.12.006

Park, C. R., Campbell, A. M., Woodson, J. C., Smith, T. P., Fleshner, M., \& Diamond, D. M. (2006). Permissive influence of stress in the expression of a U-shaped relationship between serum corticosterone levels and spatial memory errors in rats. Dose-response : a publication of International Hormesis Society, 4(1), 55-74. doi:10.2203/doseresponse.004.01.005.Park

Puig, M. V., Antzoulatos, E. G., \& Miller, E. K. (2014). Prefrontal dopamine in associative learning and memory. Neuroscience, 282, 217-229. doi:10.1016/j.neuroscience.2014.09.026

Puig, M. V., \& Miller, E. K. (2015). Neural Substrates of Dopamine D2 Receptor Modulated Executive Functions in the Monkey Prefrontal Cortex. Cereb Cortex, 25(9), 2980-2987. doi:10.1093/cercor/bhu096 
Rana, S., Nam, H., Glover, M. E., Akil, H., Watson, S. J., Clinton, S. M., \& Kerman, I. A. (2016). Protective effects of chronic mild stress during adolescence in the low-novelty responder rat. Stress, 19(1), 133-138. doi:10.3109/10253890.2015.1108304

Reincke, S. A., \& Hanganu-Opatz, I. L. (2017). Early-life stress impairs recognition memory and perturbs the functional maturation of prefrontal-hippocampal-perirhinal networks. Sci Rep, 7, 42042. doi:10.1038/srep42042

Rowland, N. E. (2007). Food or fluid restriction in common laboratory animals: balancing welfare considerations with scientific inquiry. Comp Med, 57(2), 149-160. Retrieved from https://www.ncbi.nlm.nih.gov/pubmed/17536615

Silk, J. S., Vanderbilt-Adriance, E., Shaw, D. S., Forbes, E. E., Whalen, D. J., Ryan, N. D., \& Dahl, R. E. (2007). Resilience among children and adolescents at risk for depression: Mediation and moderation across social and neurobiological contexts. Dev Psychopathol, 19(3), 841-865. doi:10.1017/S0954579407000417

Spencer-Segal, J. L., \& Akil, H. (2019). Glucocorticoids and resilience. Horm Behav, 111, 131134. doi:10.1016/j.yhbeh.2018.11.005

Srinivasan, S., Shariff, M., \& Bartlett, S. E. (2013). The role of the glucocorticoids in developing resilience to stress and addiction. Front Psychiatry, 4, 68. doi:10.3389/fpsyt.2013.00068

Teicher, M. H., \& Samson, J. A. (2013). Childhood maltreatment and psychopathology: A case for ecophenotypic variants as clinically and neurobiologically distinct subtypes. Am J Psychiatry, 170(10), 1114-1133. doi:10.1176/appi.ajp.2013.12070957

Teicher, M. H., Samson, J. A., Anderson, C. M., \& Ohashi, K. (2016). The effects of childhood maltreatment on brain structure, function and connectivity. Nat Rev Neurosci, 17(10), 652-666. doi:10.1038/nrn.2016.111 
Tractenberg, S. G., Levandowski, M. L., de Azeredo, L. A., Orso, R., Roithmann, L. G., Hoffmann, E. S., . . Grassi-Oliveira, R. (2016). An overview of maternal separation effects on behavioural outcomes in mice: Evidence from a four-stage methodological systematic review. Neurosci Biobehav Rev, 68, 489-503. doi:10.1016/j.neubiorev.2016.06.021

Umemori, J., Takao, K., Koshimizu, H., Hattori, S., Furuse, T., Wakana, S., \& Miyakawa, T. (2013). ENU-mutagenesis mice with a non-synonymous mutation in Grin1 exhibit abnormal anxiety-like behaviors, impaired fear memory, and decreased acoustic startle response. BMC Res Notes, 6, 203. doi:10.1186/1756-0500-6-203

Valentim, A. M., Olsson, I. A., \& Antunes, L. M. (2013). The anaesthetic combination of ketamine/midazolam does not alter the acquisition of spatial and motor tasks in adult mice. Lab Anim, 47(1), 19-25. doi:10.1258/la.2012.011179

Valladolid-Acebes, I., Stucchi, P., Cano, V., Fernandez-Alfonso, M. S., Merino, B., GilOrtega, M., . . . Del Olmo, N. (2011). High-fat diets impair spatial learning in the radialarm maze in mice. Neurobiol Learn Mem, 95(1), 80-85. doi:10.1016/j.nlm.2010.11.007

van der Kooij, M. A., Grosse, J., Zanoletti, O., Papilloud, A., \& Sandi, C. (2015). The effects of stress during early postnatal periods on behavior and hippocampal neuroplasticity markers in adult male mice. Neuroscience, 311, 508-518. doi:10.1016/j.neuroscience.2015.10.058

Vijayraghavan, S., Wang, M., Birnbaum, S. G., Williams, G. V., \& Arnsten, A. F. (2007). Inverted-U dopamine D1 receptor actions on prefrontal neurons engaged in working memory. Nat Neurosci, 10(3), 376-384. doi:10.1038/nn1846

Viola, T. W., Wearick-Silva, L. E., Creutzberg, K. C., Kestering-Ferreira, E., Orso, R., Centeno-Silva, A., . . . Grassi-Oliveira, R. (2019). Postnatal impoverished housing impairs adolescent risk-assessment and increases risk-taking: A sex-specific effect 
associated with histone epigenetic regulation of Crfr1 in the medial prefrontal cortex. Psychoneuroendocrinology, 99, 8-19. doi:10.1016/j.psyneuen.2018.08.032

Wang, L., Jiao, J., \& Dulawa, S. C. (2011). Infant maternal separation impairs adult cognitive performance in BALB/cJ mice. Psychopharmacology (Berl), 216(2), 207-218. doi:10.1007/s00213-011-2209-4

Wearick-Silva, L. E., Orso, R., Martins, L. A., Creutzberg, K. C., Centeno-Silva, A., Xavier, L. L., . . Mestriner, R. G. (2019). Dual influences of early life stress induced by limited bedding on walking adaptability and Bdnf/TrkB and Drd1/Drd2 gene expression in different mouse brain regions. Behav Brain Res, 359, 66-72. Retrieved from https://www.ncbi.nlm.nih.gov/pubmed/30347225.doi:10.1016/j.bbr.2018.10.025

Wickens, J. R., Horvitz, J. C., Costa, R. M., \& Killcross, S. (2007). Dopaminergic mechanisms in actions and habits. J Neurosci, 27(31), 8181-8183. doi:10.1523/JNEUROSCI.167107.2007

Williams, G. V., \& Castner, S. A. (2006). Under the curve: critical issues for elucidating D1 receptor function in working memory. Neuroscience, 139(1), 263-276. doi:10.1016/j.neuroscience.2005.09.028

Winstanley, C. A., \& Floresco, S. B. (2016). Deciphering Decision Making: Variation in Animal Models of Effort- and Uncertainty-Based Choice Reveals Distinct Neural Circuitries Underlying Core Cognitive Processes. J Neurosci, 36(48), 12069-12079. doi:10.1523/JNEUROSCI.1713-16.2016

Yadav, R., Hillman, B. G., Gupta, S. C., Suryavanshi, P., Bhatt, J. M., Pavuluri, R., . . Dravid, S. M. (2013). Deletion of glutamate delta-1 receptor in mouse leads to enhanced working memory and deficit in fear conditioning. PLoS One, 8(4), e60785. doi:10.1371/journal.pone.0060785 
Yehuda, R., Flory, J. D., Pratchett, L. C., Buxbaum, J., Ising, M., \& Holsboer, F. (2010). Putative biological mechanisms for the association between early life adversity and the subsequent development of PTSD. Psychopharmacology (Berl), 212(3), 405-417. doi:10.1007/s00213-010-1969-6

Yehuda, R., Flory, J. D., Southwick, S., \& Charney, D. S. (2006). Developing an agenda for translational studies of resilience and vulnerability following trauma exposure. Ann $N$ Y Acad Sci, 1071, 379-396. doi:10.1196/annals.1364.028

Yoon, T., Okada, J., Jung, M. W., \& Kim, J. J. (2008). Prefrontal cortex and hippocampus subserve different components of working memory in rats. Learn Mem, 15(3), 97-105. Retrieved from $\quad$ https://www.ncbi.nlm.nih.gov/pubmed/18285468. doi:10.1101/lm.850808

Zohar, J., Yahalom, H., Kozlovsky, N., Cwikel-Hamzany, S., Matar, M. A., Kaplan, Z., . . . Cohen, H. (2011). High dose hydrocortisone immediately after trauma may alter the trajectory of PTSD: interplay between clinical and animal studies. Eur Neuropsychopharmacol, 21(11), 796-809. doi:10.1016/j.euroneuro.2011.06.001 